\title{
Monolithic Earthen Shells Digital Fabrication: Hybrid Workflow
}

\author{
Maite Bravo $^{1}$ and Stephanie Chaltiel ${ }^{2}$ \\ ${ }^{1}$ Institute of Advanced Architecture of Catalunya IAAC \\ maite.bravo@iaac.net \\ ${ }^{2}$ Institute of Advanced Architecture of Catalunya IAAC, University Polytechnic of Catalunya \\ UPC \\ stephanie.chaltiel@iaac.net
}

\begin{abstract}
The digital fabrication process of monolithic shell structures is presenting some challenges related to the interface between computational design and fabrication techniques, such as the translation from the digital to the physical when a definite materiality appears during the final phase of the fabrication process, which is typically approached as a linear and predetermined sequence, most often not allowing any kind of adjustments or rectifications. This stage proves to be critical for monolithic shells, because their integrity relies on the continuity of the construction phases for its stability and durability. This paper exposes the implementation process required for new computational and physical methods for monolithic shells construction using digital fabrication techniques combined with skilled/non-monotonous manual craft. It proposes an additive manufacturing deposition process for paste clay mixes with the potential of embedding a certain level of interactivity between the fabricator and the materialized model during the fabrication process, to allow for real time adjustments or corrections. Two case studies are featured, detailing the steps required to take advantage of the flow involving manual craft, digital modeling, and digital fabrication tools. These methods aim at renewing shells construction processes, in terms of the time it takes to build them, the optimization of materials and labor, and the feasibility of the dweller to be involved during different construction phases.
\end{abstract}

Keywords: Monolithic Shells, Robotic Fabrication, Digital Craft, Earth Architecture. 


\section{Mud shell construction}

Earth construction for monolithic shells can be found in ancient traditions [1], with some strong references in the Nubian vaults (Sudan), the Harran Beehive Houses (Turkey), and the Musgum mud huts (Cameroon), among others [2]. These modest but relevant examples are usually constructed with or without formwork with small lumps of sticky clay thrown by hand in layers. A similar and singular earth construction method derived from the "Wattle and daub" or "Torchi" [3] composed of vertical wattles, woven branches forming a support surface, and daubed with clay or mud to make-up an assembly of interlocked materials. Through the use of digital design and robotic fabrication, these traditional techniques could be systematized to respond to local conditions, and to embed additional optimization criteria.

The stability for the construction of monolithic shell structures is typically provided by a temporary formwork until the material reaches sufficient strength, which exhibits several challenges, such as the extensive use of labor and materials. As a result, fabric formwork has been explored [4] because of its ability to provide a temporary support that can be adjusted and offers greater freedom for forms, a controlled smooth finish, easy removal techniques, and an overall inexpensive solution. Fabric formwork in concrete include projects such as Mark West (C.A.S.T) [5], Matsys [6], and Cloud 9 STGILAT pavilion [7], among many others.

Additive manufacturing is being currently implemented at diverse scales and techniques in earth construction, where robotic fabrication is taking a relevant place with two main strategies: extrusion and spraying. Extrusion is conducted through a nozzle by which the paste like material is deposited, achieving complex geometries, but heavily restricted to the size and characteristics of the deposition apparatus. Examples include Co-de-it, inFORMed clay matter [8]; G Code Clay [9]; Wasp [10]; and Mataerial/Pylos/On-Site-Robotics [11]. The spraying technique deposits material through a nozzle under pressure piped to a mix of cement or clay that is simultaneous drying while printing in the air. Spraying has a long history using shotcrete (both wet and dry concrete mix) [12], and it's widely used in a variety of construction applications including civil works, pools, and buildings.

This paper explores the implementation of digital fabrication for monolithic shells using mud spray techniques. 


\section{Hybrid workflow for mud shell additive manufacturing}

The proposed fabrication method for mud monolithic shells are based on a collaborative framework that merges manual actions carefully coordinated with digital and robotic dynamics. The organization of a critical sequence must be properly formulated, so an iterative workflow in calibrated loops that are detailed below.

\subsection{Placement, consolidation, and finishing loops}

Loop 1- PLACEMENT: The initial manual formwork set-up is composed of a wooden baseboard with holes supporting groups of bending rods to create supporting arches (typically 3 to 4 outer arches and 1 to 3 middle nerves), from where a stretched fabric is secured. The scan \#1 of the fabric formwork uses high-resolution photography and Agisoft image processing software, then exported into a CAD environment (Rhino 3d) to generate a $3 \mathrm{~d}$ mesh that is simplified by reducing the number of divisions or redrawing of main curves. Afterwards, the optimization \#1 includes different structural simulations using Karamba, a parametric structural engineering tool, to display analysis of tension or compression areas, or stress lines for the potential placement of additional reinforcement. Robotic trajectories are designed in Kuka PRC (digital processes) with settings based on previous tests, but require constant readjustment on the trajectory and velocity (distance and angle to the structure) to achieve a homogeneous deposition and a proper mix adhesion to the fabric formwork. The material spray \#1 starts with the manual clay preparation that includes soil test, mixing, and feeding the sprayer. The first material layer is a liquid mix "barbotine" (clay and water), that is sprayed on top of the fabric to facilitate an optimum adhesion for the following layers.

Loop 2 - CONSOLIDATION: This stage includes the material placement in several layers, therefore repeated iteratively. The scan \#2 is crucial, because it allows keeping records of the morphology in progress. The optimization \#2 seeks a minimum use of material (thickness) for maximum strength, simulations show the shell regions defined by displacement criteria, or some expected deformations of the shell in progress such as the sagging on both sides of the ribs. However, these optimizations are only approximations, because they are based in Karamba on the properties of concrete, as clay is not yet available in the 
software. Robotic trajectories must be constantly recalibrated and reinserted into the Kuka PRC software (trajectory, thickness, etc.). The middle layers contain clay, sand and natural fibers (that add significant shell thickness while being light in weight), a mix prepared in a large bucket and stirred by hand. A custom-made spray tool nozzle allows different perforations, and must be calibrated in terms of the angle, distance, and speed of each deposition.

Loop 3 - FINISHES: Includes the embedment of special finishes (additives), curing time, thickness checkup, final coating, and the removal of temporary formwork.

This precise protocol needs to be respected to ensure the solidity of the resulting structure.

\subsection{Case studies}

Recent academic experiments investigate new methods and provide a more realistic set up towards patented fabrication techniques. Two case studies presented in this paper propose a complete strategy for the digital fabrication of mud shells reaching a height up to $2.0 \mathrm{~m}$.

\section{Case Study 1:}

May 2016. 25 hrs. seminar. First year master students. IAAC, Barcelona.

Seminar: "Phriends for Shells" ("Phriends" was defined as the safe interaction between people and robots during the fabrication progress).

Participants: 23

Tutors: Author 1, D. Stanejovic (robotic expert), Y. Mendez (assistant).

Five earthen shells of $1.5 \mathrm{~m} \times 1.5 \mathrm{~m} \times 1 \mathrm{~m}$ (height) with perforations were built during this 4 weeks seminar.

Loop 1- PLACEMENT: Bending rods in clusters of 2 to 3 members bundled together with a rope (Fig. 1) were used as supporting arches where the stretched lycra was secured. Openings with perforations were defined with laser cut rings and triangles that were used to create a temporary formwork that was removed afterwards. 


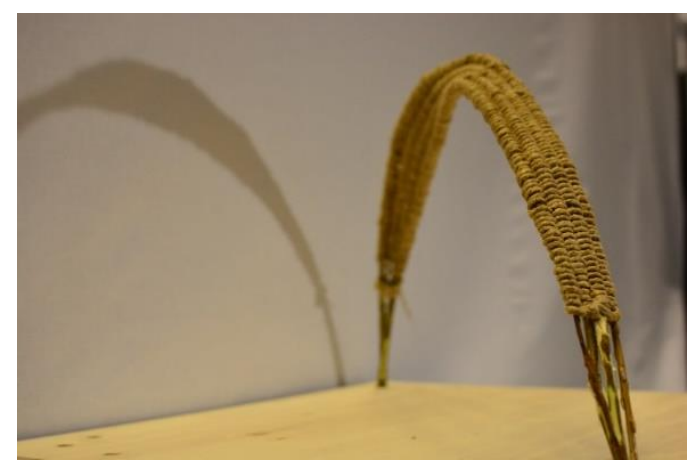

Fig. 1. Cluster of 3 bending reeds bundled together. [Photos by the participants]. 2016.

The scan \#1 was done with a Sony camera on manual mode, and to facilitate the accuracy of the scan, the lycra fabric required contrasting marks done manually (Fig. 2).
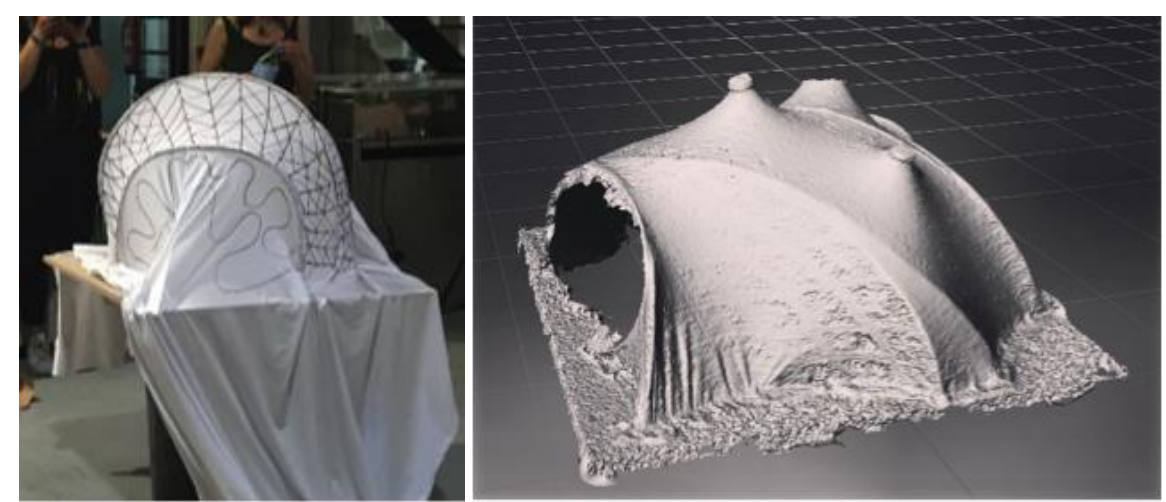

Fig. 2. 3D scanning\#1 and rebuild mesh on Rhino 3D. Earthen Shells workshop. 2017.

The optimization \#1 in Karamba simulations analysis (Fig. 3) revealed that the inclusion of perforations (triangles or circles), position, density and proximity to the main nerves could be parametrized within the shell's geometry and could minimize the amount of tension areas. The study of the perforations tested in both digital and physical models appeared to lead to the most successful designs.

The material spray \#1 includes a first layer containing $1 \mathrm{U}$ clay $+2 \mathrm{U}$ water. The deposition tool was a Wagner heavy paint sprayer Flexio 590 HVLP with external air compressor (kit part). The deposition was at a 45 -degree angle, at $30 \mathrm{~cm}$ from the surface, and at a speed of $2 \mathrm{~m} / \mathrm{s}$. 

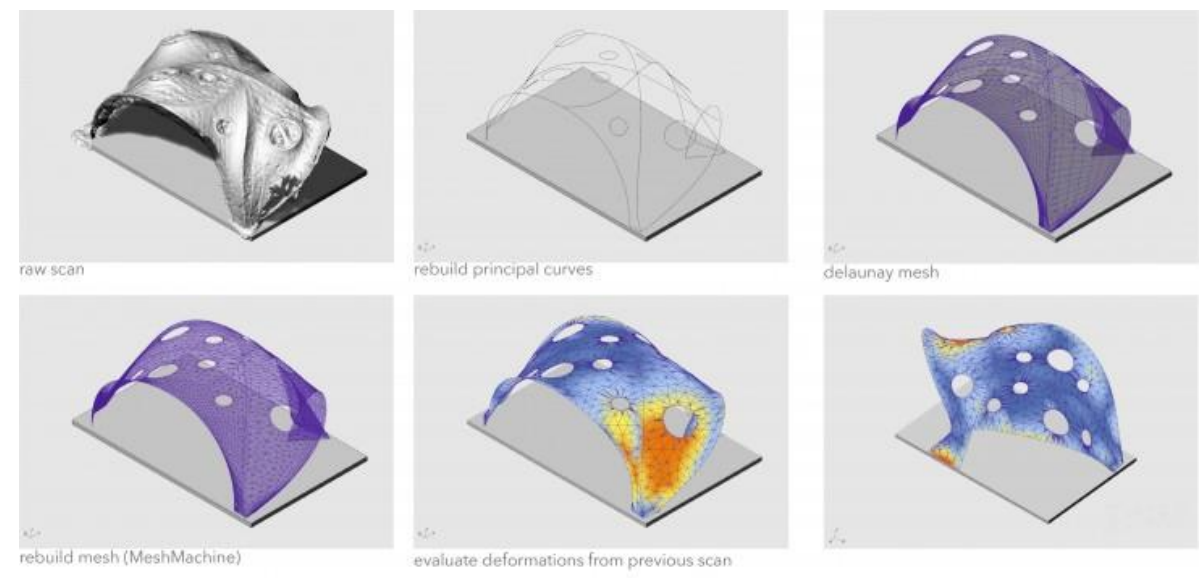

Fig. 3. Initial $3 d$ scan, rebuild mesh, and deformations evaluation in Karamba simulation [Group 1, Iaac seminar, May 2016].

Loop 2 to 6 -CONSOLIDATION A: Five layers were sprayed (Fig. 4) in iterative loops. The scanning process was time consuming because it required recalibrating the trajectory of the applied spray each time. The optimization \#2 in Karamba revealed some considerable deformations around the perforations areas while being applied, because of the considerable weight of the sprayed clay mixes. A reevaluation of the initial optimization considered a variation in thickness; therefore if some areas appeared fragile after a fresh layer was applied, the deposition was stopped and corrected. The loop was repeated four times, and sequential scans revealed that the shape was changing dramatically during the process due to the weight of the wet clay mix while being sprayed. It was observed that when too much material was applied, a risk of collapsing of the shell or of some its parts appeared. Robotic trajectories needed to be constantly recalibrated from the default 45 degrees and 30 $\mathrm{cm}$ distance to the surface, required constant readjustment and were reinserted into the Kuka PRC software. The material spray with middle layers contained $3 U$ fibers $+2 U$ clay $+2 U$ sand $+2 U$ water, with a manual concrete sprayer "Sablon" with a cone of $30 \mathrm{~cm}$ that needed to be refilled regularly. The angle was 20 degrees to the surface normal (because greater angles provoked falling of the mix out of the cone), at a distance of $20 \mathrm{~cm}$ from the surface, reaching a speed of $5 \mathrm{~m} / \mathrm{s}$. 


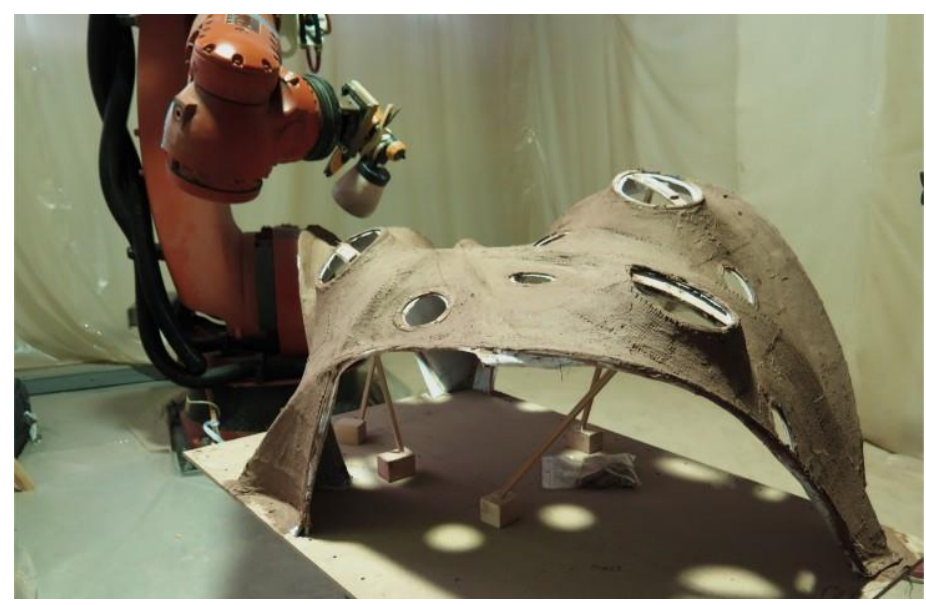

Fig. 4. Kuka robot with end effector: Wagner heavy paint sprayer applying the clay mix of the first layers on the earthen shell. Iaac seminar May 2016. [Photos by the seminar's participants].

Loop 7 - FINISHES: A last layer was robotically sprayed with a mix that included $1 \mathrm{U}$ clay $+1 \mathrm{U}$ sand (water was not needed) $+2 \mathrm{U}$ stabilizers to prevent cracks and to provide better waterproofing (highly viscous cactus solution by boiling thin slices of cactus and stirring until it turned into a highly viscous mix). The deposition tool was set at a speed of $2 \mathrm{~m} / \mathrm{s}$; a 45-degree angle; and a distance of $30 \mathrm{~cm}$ from the shell. The shells reached at least a total $2 \mathrm{~cm}$ in overall thickness. The curing time was a total of 3 full days to complete 5 coatings (working indoors during summer at 25 degrees C). Drying time was at least around 4 to 5 hours in between each spray layer, and the lycra fabric and bending rods were removed manually.

Case Study 2:

May 2017; 25 hours seminar, first year master students. IAAC, Barcelona.

Seminar: "Earthen Shells Digital and Manual Fabrication."

Participants: 13

Tutors: Author 1, A. Ibrahim, N. Elgewely, A.Chronis, K. Dhanabalan, K. Singh Chadha.

This seminar proposes the design and fabrication of three earthen shells with some $3 \mathrm{~d}$ texturing and perforations with embedded natural resin translucent modules, reaching $1.5 \mathrm{~m} \times 1.5 \mathrm{~m} \times 1.0 \mathrm{~m}$ in size (Fig. $5)$. 


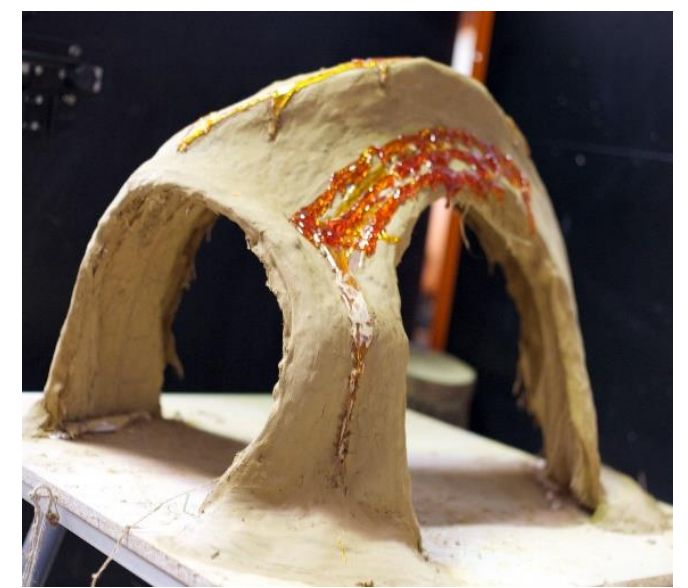
017

Loop 1- PLACEMENT: The scan \#1 used two Parrot AR Drones, 2.0 Elite Edition (about 70 pictures), controlled from smart phones and tablets by open codes that can be modified by non-experts. Optimization \#1 was run with Karamba simulations with tension and compression regions of the shell paired with stress lines. The material spray \#1 included a first layer only contains $2 \mathrm{U}$ water $+1 \mathrm{U}$ clay. The robotic spray was set at a constant distance of $3 \mathrm{~cm}$ from the shell to the end of the nozzle, at a fixed angle of 45 degrees to the surface shell normal at all points.

Loop 2 - CONSOLIDATION A: The scan \#2 was performed, and the optimization in Karamba highlighted tension and compression areas, revealing a considerable shell deformation during the fabrication process, which lead to evaluate the inclusion of a real time recalibration tool with preliminary augmented reality experiments (Fig. 6), that were tested to explore the physical structure projecting the virtual model simulating the thickness variations, or by changing the location of the perforations. The material spray \#2 includes applying strips of jute fabric in between robotically sprayed clay mix to reinforce the structure, and to allow a subtle placement of non-clay modules, or the translucent embedded openings. Once the shell surfaces are modeled in Rhino 3d, the geometries are unrolled to facilitate the design of a laser cut cutting pattern on the jute fabric. The middle layers contain clay, sand and fibers, deposited with a concrete hand sprayer placed as the "end effector 
"of the robotic arm. The trajectory is set to adapt to the shells form by spraying at a velocity of $2 \mathrm{~m} / \mathrm{s}$, in horizontal straight lines (contours), at an average $20 \mathrm{~cm}$ from the surface (Fig. 7).

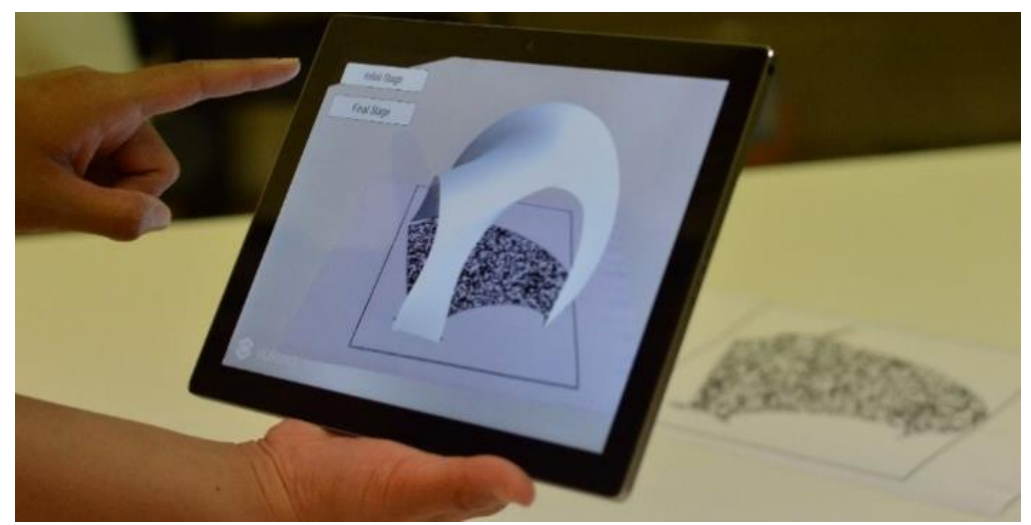

Fig. 6. Augmented reality tests. 2017. Earthen Shells'workshop..

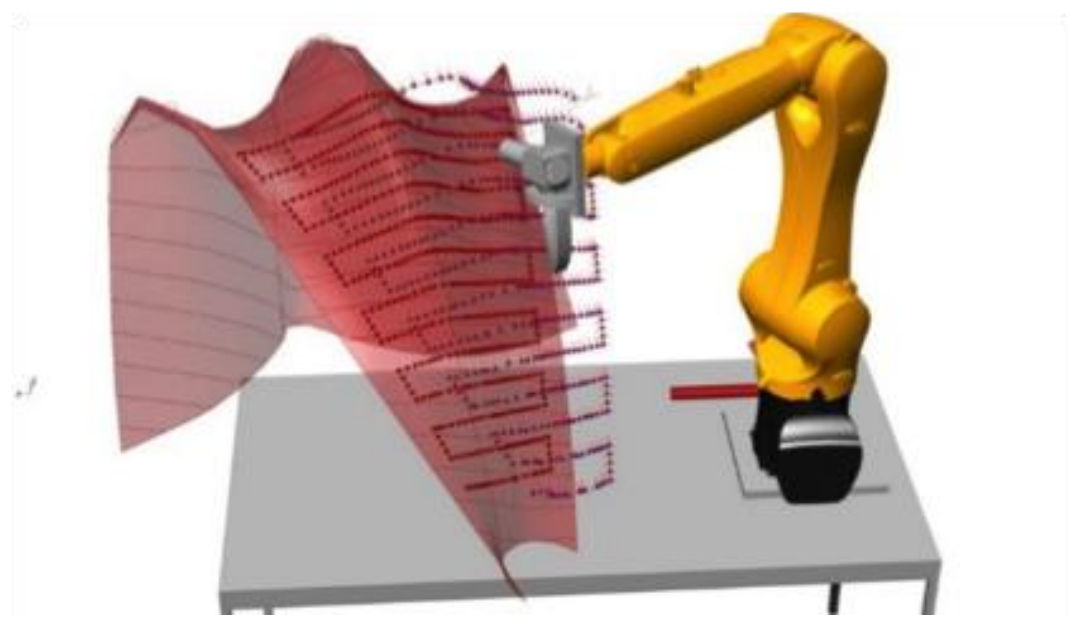

Fig. 7. Continuous robotic spraying simulation in Kuka prc. Smart Geometry Cluster "Mud, Textile and Robots", Gothenburg 2016.

Loop 3 - CONSOLIDATION B: The scan \#3 as the structure was already fixed on the robotic turning table some photos taken from the robot. From this scan, the structure appeared to have sagged on each part of the supporting arches. The optimization \#3 revealed shell areas working primarily under tension or under compression, that were as- 
signed thickness variations (Fig. 8). Compression areas were given the thickness (T), while tension areas were given the thickness (T-1). The thickness variation is achieved by changing the number of robotically sprayed layers. In addition, the middle layers of sprayed fibrous mix had a thickness of $1 \mathrm{~cm}$, while the sprayed layer of clay sand and water was $2 \mathrm{~mm}$.

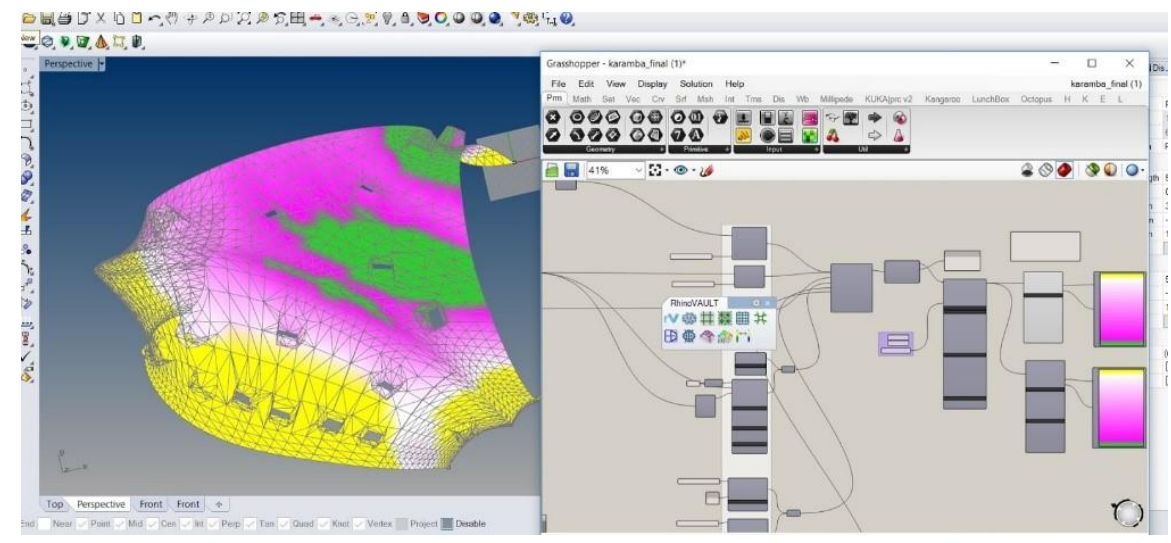

Fig. 8. Karamba simulation highlighting areas working in compression or in tension "Earthen Shells Workshop". 2017.

Loop 4 - FINISH A: The material Spray \#3 for the upper layer included $1 \mathrm{U}$ clay $+1 \mathrm{U}$ sand mix $+1 \mathrm{U}$ water $+1 \mathrm{U}$ olive oil (a natural stabilizer for waterproofing and to avoid cracks). The deposition Tool was a Wagner spray paint was used by hand or attached to the robot for the upper layers application, $25 \mathrm{~cm}$ from the surface angle of 45 degrees to the surface normal, and velocity of deposition $2 \mathrm{~m} / \mathrm{s}$. Total thickness was $3 \mathrm{~cm}$, and curing time was only 2 hours (outdoor hot and dry weather at 30 degrees $\mathrm{C}$ ). Curing time checkup included knocking in different areas to verify that the structure was stable, allowing the manual removal of the temporary formwork.

Loop 5 - FINISH B: This layer includes some natural resin modules robotically poured while hot (Fig. 9). The readjustment of the velocity, pouring angle, and material temperature, allowed different textures and effects to be explored. 


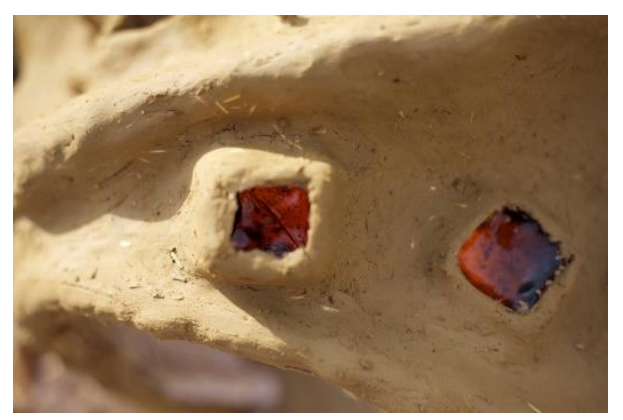

Fig. 9. Natural resin robotic pouring. Earthen Shells'workshop 2017.

\section{Next steps for Mud Shell Digital Fabrication:}

This paper proposed a framework for additive manufacturing spray protocol for the construction of earthen monolithic shells that integrates manual with automated processes for novel collaborative techniques. These collaborations are not just conceived as isolated chain of tasks, but a process where manual manufacturing responds to and creates the demand for specific robotic actions in an integrated feedback loop, happening according to a precise phasing. These protocols have been tested in several instances, and are still being continuously improved and enhanced, and hope to be implemented at full scale in the near future.

The potential to execute full-scale constructions depends also on several specific factors drawn from the case studies presented in this paper, such as greater automatization, reliable material tests, continuous adjustment of reinforcement, real time control, among others.

An increased or full degree of automatization for certain rather laborious and repetitive tasks must be considered, such as the stirring of the heavy clay mix so that to achieve more homogeneous mix. Although the material proportions in the experiments were set according to previous physical tests results, the mixes were evaluated at each application only by hand (with the criteria of sticking to the previous layer), therefore exposing the limitation that properties can't be constant in terms of air and water content. Future experiments require a more scientific approach to avoid such variations in the mix consistency (clay or sand types) and an increased control in the material characteristics (air content, humidity, or workability). The proposed method includes two main families of manual actions collaborating with robotic spraying: 
deposition of the reinforcement strips, and application of the middle and last coating by hand. Some aspects of this fabrication stage are done by hand, such as the reinforcement strips placement according to strength and weakness of the overall structure in progress, but the location of the reinforcement should be further rationalized, so that it could be layered robotically involving the fabrication of a custom-made end effector.

Full-scale implementation must resolve some other limitations that include: testing for changing material properties (as material can be locally collected); structural engineering analysis of different shell morphologies (load, torsion, shear) with load tests according to the ratio height/thickness or according to variations on material thicknesses; the incidence of local conditions with live sensors to collect environmental data (weather, humidity, temperature, etc.), and optimization protocols to respond to local conditions (such as wind).

Varying the location and density of reinforcements could fully integrate the optimization analysis stage by identifying stress lines (Fig. 10) to add localized strength according to simulations and physical realtime distortion prone areas (Fig. 11). Stress lines-reinforcement can include adding thickness in the clay poor, or incorporating an additional material such as the applied single layer of jute strips, bamboo strands, sugar cane, reeds, amongst others as lost formworks.

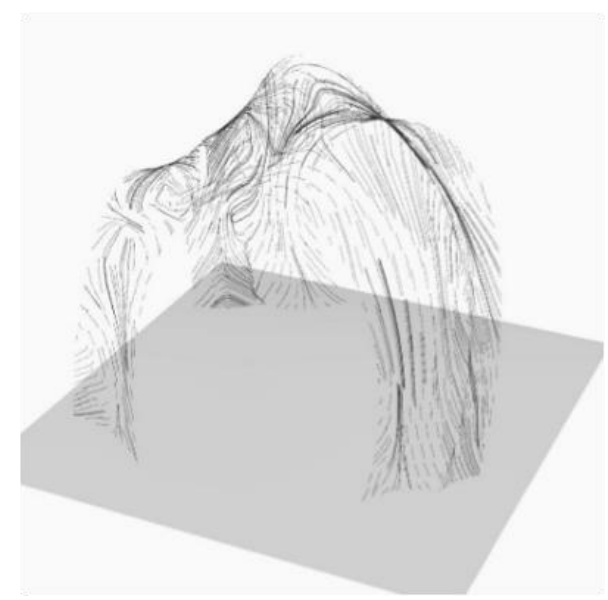

Fig. 10. Stress lines optimization in Karamba. Earthen Shells workshop. 2017.

Finishing by hand certain inaccessible and limited areas might be necessary to increase the viability of the shell and its resilience against 
the rain (i.e around the perforations), and for ongoing maintenance reasons.

The implementation of real time control using augmented reality could provide an increased decision-making power for the builder, revealing an unexpected degree of freedom and creativity such processes could bring to monolithic shell construction. Real time $3 \mathrm{~d}$ scanning available in other industries will improve the technique by allowing manual workers and robotic actions controller to adapt constantly to the structure's deformation and non-predictable changes in shape in specific regions of the shell (such in case study 2). Or the possibility of implementing remote control in real time could lead to new horizons in construction protocols, if external expert feedback can be implemented for remote locations while under construction.

These explorations show how the successive and simultaneous actions performed collaboratively by robots and humans would not only engender new workflows for mud shell construction, but propose a fruitful dialogue between computational techniques and revisited traditional crafts, that when implemented at full-scale could open an unexplored and innovative agenda for additive manufacturing.

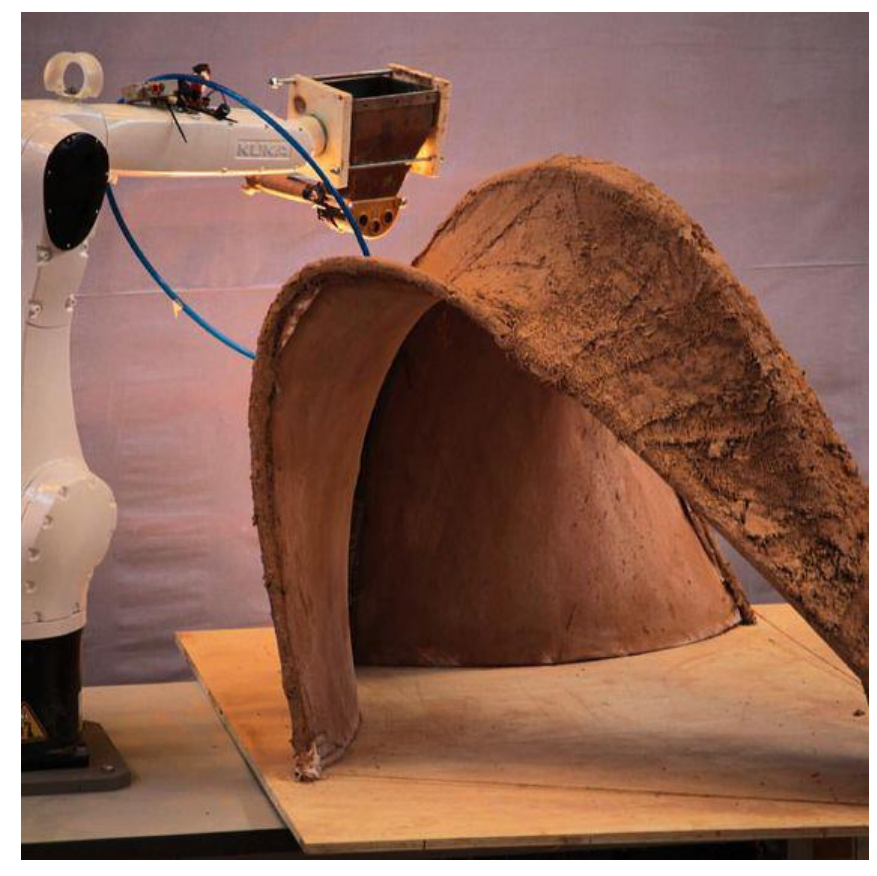




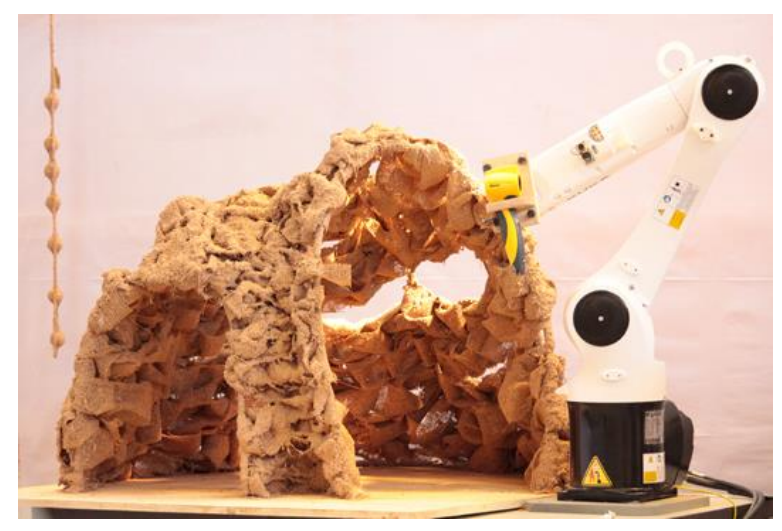

Fig. 11. Robotically sprayed mud shell with temporary fabric formwork (top) and weaved jute fabric as lost formwork (bottom). Smart Geometry 2016. Gothenburg. Photo Andrea Quetara.

\section{Acknowledgments}

This research forms part of the Marie Skłodowska-Curie Actions, ITN Innochain, grant number \#642877.

\section{References}

1. Stulz, Roland., Kiran. Mukerji, and Schweizerische Kontaktstelle für Angepasste Technik am ILE. Appropriate Building Materials: A Catalogue of Potential Solutions. SKAT, 1993.

2. Niroumand, Hamed, M.F.M. Zain, and Maslina Jamil. Assessing of Critical Parameters on Earth Architecture and Earth Buildings as a Vernacular and Sustainable Architecture in Various Countries. Procedia - Social and Behavioral Sciences 89 (October 2013): 248-60. doi:10.1016/j.sbspro.2013.08.843.

3. Rael, R. (2009). Earth architecture. Princeton Architectural Press.

4. Diederik Veenendaal*, Mark West, and Philippe Block. "History and Overview of Fabric Formwork: Using Fabrics for Concrete Casting." DOI: 10.1002/suco.201100014, 2011. doi:10.1002/suco.201100014.

5. West, Mark. C.A.S.T. The Centre for Architectural Structures and Technology (CAST), Faculty of Architecture, University of Manitoba.

6. Matsys, flexible formwork, http://matsysdesign.com/tag/flexible-formwork/ XXX, last accessed 2017/05/19.

7. Cloud 9, STGILAT pavilion, 2015, http://arcaute-zaragoza.com/stgilat.html, last accessed 2017/05/19.

8. "Informed Matter." http://www.co-de-it.com/wordpress/category/informed-matter, last accessed 2017/05/19.

9. "GCODE.Clay | Emerging Objects." http://www.emergingobjects.com/project/gcodeclay/. Accessed June 11, 2017.

10. “3D Printers Manufacturer, WASP.” Accessed June 11, 2017. http://www.wasproject.it/w/en/.

11. Iaac, Large Scale 3d printing: Mataerial, Pylos, On-Site-Robotics. Accessed June 11, 2017. https://iaac.net/research-projects/

12. Gedeon, Gilbert. P.E., "Introduction to Shotcrete Applications", 31 January 1993. 